\title{
A note on the strong maximal operator on $\mathbb{R}^{n}$
}

\author{
by \\ Jiecheng Chen and Xiangrong Zhu (Hangzhou)
}

Abstract. We prove that for $f \in L \ln ^{+} L\left(\mathbb{R}^{n}\right)$ with compact support, there is a $g \in L \ln ^{+} L\left(\mathbb{R}^{n}\right)$ such that (a) $g$ and $f$ are equidistributed, (b) $M_{S}(g) \in L^{1}(E)$ for any measurable set $E$ of finite measure.

1. Introduction. For a function $f \in L_{\mathrm{loc}}\left(\mathbb{R}^{n}\right)$, its Hardy-Littlewood maximal function is defined by

$$
M(f)(x)=\sup _{Q \ni x} \frac{1}{|Q|} \int_{Q}|f(y)| d y,
$$

where $Q$ is a cube with sides parallel to the coordinate axes, and its strong maximal function is defined by

$$
M_{S}(f)(x)=\sup _{P \ni x} \frac{1}{|P|} \int_{P}|f(y)| d y,
$$

where $P$ is a rectangle with sides parallel to the coordinate axes. In addition, let $M^{*}(f)(x)=M_{n} \circ \cdots \circ M_{1}(f)(x)$, where $M_{j}$ is the Hardy-Littlewood maximal operator on $\mathbb{R}^{1}$ acting on the $j$ th coordinate $x_{j}$.

It is well known that for $f$ with compact support,

- $M(f) \in L^{1}(E)$ for any measurable set $E$ of finite measure $\Leftrightarrow f \in$ $L \ln ^{+} L\left(\mathbb{R}^{n}\right)$ (see Stein [5]).

- $M^{*}(f) \in L^{1}(E)$ for any measurable set $E$ of finite measure $\Leftrightarrow f \in$ $L\left(\ln ^{+} L\right)^{n}\left(\mathbb{R}^{n}\right)$ (see Jessen-Marcinkiewicz-Zygmund [4] and FavaGatto-Gutiérrez [2]).

- $f \in L\left(\ln ^{+} L\right)^{n}\left(\mathbb{R}^{n}\right) \Rightarrow M_{S}(f) \in L^{1}(E)$ for any measurable set $E$ of finite measure, because $M_{S}(f) \leq M^{*}(f)$.

It was conjectured that for $f \in L\left(\ln ^{+} L\right)^{n-1}\left(\mathbb{R}^{n}\right), M_{S}(f) \in L^{1}(E)$ for any measurable set $E$ of finite measure $\Rightarrow f \in L\left(\ln ^{+} L\right)^{n}\left(\mathbb{R}^{n}\right)$ (see [2]). In [1]

2000 Mathematics Subject Classification: Primary 42B25; Secondary $42 \mathrm{~B} 35$.

Key words and phrases: strong maximal operator, Zygmund class, local integrability.

Research supported by 973-project of China (G1999075105), RFDP (20030335019) and ZJNSFC (RC97017). 
and [3], Bagby and Gomez independently proved that there are many functions $f \in L \ln ^{+} L\left(\mathbb{R}^{2}\right)$ such that $M_{S}(f) \in L^{1}(E)$ for any measurable set $E$ of finite measure.

In this paper, in a different way which can be easily applied to higher dimensions, we shall prove that the conjecture is also not true for $n>2$. An interesting thing is that we do not need $f \in L\left(\ln ^{+} L\right)^{n-1}\left(\mathbb{R}^{n}\right)$.

TheOREM 1. For $f \in L \ln ^{+} L\left(\mathbb{R}^{n}\right)$ with compact support, there is a $g \in$ $L \ln ^{+} L\left(\mathbb{R}^{n}\right)$ such that (a) $g$ and $f$ are equidistributed, (b) $M_{S}(g) \in L^{1}(E)$ for any measurable set $E$ of finite measure.

2. Proof of the Theorem. Before proving the above theorem, we first introduce some notations and give some lemmas. Let

$$
\begin{aligned}
& A_{t}=\left\{\left(x_{1}, \ldots, x_{n}\right): \sum_{i=1}^{n} x_{i}=t\right\} \\
& D=\left\{\left(x_{1}, \ldots, x_{n}\right): \sum_{i=1}^{n} x_{i} \geq n-1, x_{i} \leq 1(i=1, \ldots, n)\right\} \\
& t(x)=\sum_{i=1}^{n} x_{i}, \quad v(x)=\mu_{n}(\{y \in D: t(y)<t(x)\}),
\end{aligned}
$$

where $\mu_{n}$ denotes the Lebesgue measure on $\mathbb{R}^{n}$. Without loss of generality, we may assume that

$$
\mu_{n}\left(\left\{x \in \mathbb{R}^{n}:|f(x)|>0\right\}\right) \leq \mu_{n}(D) .
$$

Take

$$
g(x)= \begin{cases}f^{*}(v(x)) & \text { for } x \in D, \\ 0 & \text { for } x \notin D,\end{cases}
$$

where $f^{*}$ is the rearrangement function of $f$, i.e.

$$
\begin{aligned}
& f^{*}(r)=\lambda_{f}^{-1}(r):=\inf \left\{s: \lambda_{f}(s) \leq r\right\}, \\
& \lambda_{f}(s)=\mu_{n}\left(\left\{x \in \mathbb{R}^{n}:|f(x)|>s\right\}\right),
\end{aligned}
$$

for $r, s>0$. It is not difficult to show that $f$ and $g$ have the same distribution function, i.e.

$$
\mu_{n}\left(\left\{x \in \mathbb{R}^{n}:|f(x)|>s\right\}\right)=\mu_{n}\left(\left\{x \in \mathbb{R}^{n}:|g(x)|>s\right\}\right)
$$

for all $s>0$.

Let $\widetilde{g}(s)=\sup \{g(x): t(x)=s\}$. It is easy to check that $\operatorname{supp}(\widetilde{g}) \subseteq$ $[n-1, n], g \in L \ln ^{+} L\left(\mathbb{R}^{n}\right) \Rightarrow \widetilde{g} \in L \ln ^{+} L\left(\mathbb{R}^{1}\right)$, and $\widetilde{g} \in L \ln ^{+} L\left(\mathbb{R}^{1}\right) \Rightarrow g \in$ $L \ln ^{+} L\left(\mathbb{R}^{n}\right)$ if $\mu_{n}\left(\left\{x \in \mathbb{R}^{n}:|f(x)|>0\right\}\right)>\mu_{n}(D)$. 
Lemma 2. $M_{S}(g)(x) \leq C_{n} M(\widetilde{g})(t(x))$, where $M_{S}$ is the strong maximal function operator on $\mathbb{R}^{n}$ and $M$ is the Hardy-Littlewood maximal function operator on $\mathbb{R}^{1}$.

Proof. For $x \in \mathbb{R}^{n}, t \in \mathbb{R}^{1}$, and $P=\prod_{i=1}^{n}\left[a_{i}, b_{i}\right] \ni x$, let

$$
d_{t}=\sup _{y \in P} d\left(y, A_{t}\right) .
$$

It is easy to see that if $P \cap A_{t} \neq \emptyset$, we have

$$
d_{t} \geq \frac{1}{2 \sqrt{n}}\left(\sum_{i=1}^{n} b_{i}-\sum_{i=1}^{n} a_{i}\right), \quad d_{t} \cdot \mu_{n-1}\left(A_{t} \cap P\right) \leq \mu_{n}(P) .
$$

So, we have

$$
\mu_{n-1}\left(A_{t} \cap P\right) \leq 2 \sqrt{n} \cdot \mu_{n}(P) /\left(\sum_{i=1}^{n} b_{i}-\sum_{i=1}^{n} a_{i}\right) .
$$

Now, let $e_{0}=\left(\sqrt{n^{-1}}, \ldots, \sqrt{n^{-1}}\right), L_{0}=\left(\mathbb{R}^{1} e_{0}\right)^{\perp}$, and $\mathbb{R}^{n} \ni x=r e_{0} \dot{+} z$, where $z \in L_{0}$. Noting that $P \ni x$ implies that $t(x) \in\left[\sum_{i=1}^{n} a_{i}, \sum_{i=1}^{n} b_{i}\right]$, we have

$$
\begin{aligned}
\frac{1}{\mu_{n}(P)} \int_{P} g(y) d y & =\frac{1}{\mu_{n}(P)} \int_{\mathbb{R}^{1} e_{0} \times L_{0}} \chi_{P}(x) g(x) d x \\
& =\frac{1}{\mu_{n}(P)} \iint_{\mathbb{R}^{1} e_{0}} \int_{L_{0}} \chi_{D}\left(r e_{0} \dot{+} z\right) g\left(r e_{0} \dot{+} z\right) d r d z \\
& \leq \frac{1}{\mu_{n}(P)} \int_{\sum_{i=1}^{n} a_{i} / \sqrt{n}}^{\sum_{i=1}^{n} b_{i} / \sqrt{n}} \mu_{n-1}\left(\left\{z: r e_{0} \dot{+} z \in P\right\}\right) \widetilde{g}(r \sqrt{n}) d r \\
& \leq \frac{1}{\sqrt{n} \mu_{n}(P)} \int_{\sum_{i=1}^{n} a_{i}}^{\sum_{i=1}^{n}} \mu_{n-1}\left(\left\{z: \frac{r}{\sqrt{n}} e_{0} \dot{+} z \in P\right\}\right) \widetilde{g}(r) d r \\
& =\frac{1}{\sqrt{n} \mu_{n}(P)} \int_{\sum_{i=1}^{n} b_{i}}^{a_{i}} \mu_{n-1}\left(A_{r} \cap P\right) \widetilde{g}(r) d r \\
& \leq \frac{2}{\sum_{i=1}^{n} b_{i}-\sum_{i=1}^{n} a_{i}} \int_{\sum_{i=1}^{n} a_{i}}^{a_{i}} b_{i}
\end{aligned}
$$

LEMMA 3. For $|x|>2 n$,

$$
M_{S}(g)(x) \leq C_{n}|x|^{-1}\|g\|_{1} .
$$

Proof. Without loss of generality, we may assume that $x_{1}>|x| / n$ for $|x|>2 n$, and furthermore, we may assume that $a_{1}<1$ and $a_{1}+\sum_{i=2}^{n} b_{i}>$ 
$n-1$ for $P=\prod_{i=1}^{n}\left[a_{i}, b_{i}\right]$ containing $x$ and such that $P \cap D \neq \emptyset$. Let $z=\left(1, b_{1}, \ldots, b_{n}\right)$. We have

$$
\begin{aligned}
\frac{1}{\mu_{n}(P)} \int_{P} g(y) d y & \leq \frac{1-a_{1}}{b_{1}-a_{1}} \frac{1}{\mu_{n}\left(\left[a_{1}, 1\right] \times \prod_{i=2}^{n}\left[a_{i}, b_{i}\right]\right)} \int_{\left[a_{1}, 1\right] \times \prod_{i=2}^{n}\left[a_{i}, b_{i}\right]} g(y) d y \\
& \leq \frac{1-a_{1}}{b_{1}-a_{1}} M_{S}(g)(z) \leq \frac{1-a_{1}}{b_{1}-a_{1}} C_{n} M(\widetilde{g})(t(z)) \\
& \leq \frac{1-a_{1}}{\left|x_{1}\right|-1} C_{n} \frac{\sqrt{n}}{t(z)-(n-1)}\|\widetilde{g}\|_{1} \\
& \leq C_{n}^{\prime} \frac{1}{|x|} \frac{1-\left(n-1-\sum_{i=2}^{n} b_{i}\right)}{1+\sum_{i=2}^{n} b_{i}-(n-1)}\|\widetilde{g}\|_{1} \leq C_{n} \frac{1}{|x|}\|g\|_{1} .
\end{aligned}
$$

From Lemmas $2-3$, we can easily get the Theorem.

\section{References}

[1] R. Bagby, A note on the strong maximal function, Proc. Amer. Math. Soc. 88 (1983), 648-650.

[2] N. A. Fava, E. A. Gatto and C. Gutiérrez, On the strong maximal function and Zygmund's class $L\left(\log _{+} L\right)^{n}$, Studia Math. 69 (1980), 155-158.

[3] M. E. Gomez, A counterexample for the strong maximal operator, ibid. 78 (1984), 199-212.

[4] B. Jessen, J. Marcinkiewicz and A. Zugmund, Note on the differentiability of multiple integrals, Fund. Math. 25 (1935), 217-234.

[5] E. M. Stein, Note on the class L $(\log L)$, Studia Math. 32 (1969), 305-310.

Department of Mathematics (Xixi Campus)

Zhejiang University

310028 Hangzhou, P.R. China

E-mail: jcchen@mail.hz.zj.cn 